\title{
Selective target processing: Perceptual load or distractor salience?
}

\author{
STACY ELTITI, DENISE WALLACE, and ELAINE FOX \\ University of Essex, Colchester, England
}

\begin{abstract}
Perceptual load theory (Lavie, 1995) states that participants cannot engage in focused attention when shown displays containing a low perceptual load, because attentional resources are not exhausted, whereas in high-load displays attention is always focused, because attentional resources are exhausted. An alternative "salience" hypothesis holds that the salience of distractors and not perceptual load per se determines selective attention. Three experiments were conducted to investigate the influence that target and distractor onsets and offsets have on selective processing in a standard interference task. Perceptual load theory predicts that, regardless of target or distractor presentation (onset or offset), interference from ignored distractors should occur in low-load displays only. In contrast, the salience hypothesis predicts that interference should occur when the distractor appears as an onset and would occur for distractor offsets only when the target was also an offset. Interference may even occur in highload displays if the distractor is more salient. The results supported the salience hypothesis.
\end{abstract}

\begin{abstract}
Attention is classically described as a limited-capacity mental resource, which can be allocated to various aspects of a scene to facilitate processing (e.g., Navon \& Gopher, 1979). One key question concerns whether it is possible for people to completely exclude distracting stimuli while responding to a relevant target. A variety of paradigms and theories have been developed in order to examine such selective processing mechanisms. Different answers have been provided by early selection and late selection theories. Early selection theory holds that objects are not recognized unless they receive attention. Instead, only basic physical features, such as spatial location, color, and/or orientation, are extracted and represented in parallel (e.g., Broadbent, 1958; Treisman \& Gormican, 1988). Due to a central-capacity limitation, focal attention is required to integrate features to form meaningful objects. Late selection theories, however, state that the recognition of familiar stimuli is an automatic process that does not require attention (e.g., Deutsch \& Deutsch, 1963; Duncan, 1980). In other words, attention is not a necessary prerequisite for all the stimuli in an environment to be processed to a semantic level.
\end{abstract}

The perceptual load theory developed by Lavie and Tsal (1994) provides a compromise to the debate by combining elements of both early and late selection theories. From early selection, it takes the idea that perception is a limited-capacity process, and from late selection it takes the idea that perception involves an automatic process as long as there are available attentional resources. Lavie and Tsal pointed out that most research involving

We thank Bruce Milliken and Lisa Paquet for their useful comments on this manuscript. Correspondence should be addressed to S. Eltiti, University of Essex, Department of Psychology, Winenhoe Park, Colchester CO4 3 SQ, England (e-mail: seltiti@essex.ac.uk). small set size displays, such as the classic Stroop task (Stroop, 1935) or the flanker task (B. A. Eriksen \& C. W. Eriksen, 1974) produce results in line with late selection theory. In contrast, experiments utilizing large set size displays produce results supportive of an early selection theory of attention (e.g., C. W. Eriksen \& Hoffman, 1972, 1973). The argument is that the perceptual load of the display is a necessary condition for selective attention to occur (Lavie \& Tsal, 1994). In other words, the major determinant of whether early or late selection will be observed is the perceptual load of a visual scene. According to the perceptual load theory, a low-load display will always invoke a late selection mode of attention, whereas a high-load display invokes an early selection mode of attention.

Lavie (1995) tested whether the ability to ignore distractors in a choice response task could indeed be determined by the perceptual load of the display. In a low-load condition, one target and one distractor letter appeared, whereas in a high-load condition, one target, five neutral letters, and one distractor letter appeared. As expected, incompatible distractors produced a significant interference effect only on the low-load displays, but not on the high-load displays. Lavie concluded that selective attention was indeed determined by the perceptual load of the relevant display.

Lavie and Fox (2000) replicated these findings and extended the research to demonstrate that perceptual load can also determine negative priming effects on a subsequent probe trial. In their experiments, interference and negative priming occurred only following low-load prime trials. When prime displays contained high perceptual load, visual processing became highly selective, as evidenced by the absence of both interference and negative priming effects (Lavie \& Fox, 2000). 
With the ongoing development of the perceptual load theory (Lavie, 1995, 2000; Lavie \& Fox, 2000; Lavie \& Tsal, 1994), an interesting question has emerged: Is it ever possible for selective processing to occur during low perceptual load displays? According to Lavie (1995), "early selection is both the inevitable outcome of allocating attention from a limited pool and impossible to achieve when the capacity is not exceeded" (p. 452). In other words, during a low-load display, it is simply not possible for participants to engage in focused attention, because not all of the attentional resources are utilized.

Evidence against this strong claim has, however, been provided by Paquet and Craig (1997), who reported evidence of selective target processing with a low perceptual load task. The classic flanker tasked developed by B. A. Eriksen and C. W. Eriksen (1974) was used with a letter target being flanked by either letters or digits. In order to test the extent of flanker processing, Paquet and Craig utilized the flanker validity effect, which is the finding that when there is a high correlation between the target and flanker item, reaction times (RTs) are faster than when the target appears with an uncorrelated flanker. Precues were used to engage participants in focused attention. The precue consisted of a fixation cross located at the center of the screen, which was presented at the same location as the target prior to the presentation of the trial stimuli. For the no-cue condition, the stimuli appeared without a fixation cross. All displays were classified as low perceptual load displays. During the precue condition, a reliable flanker validity effect was found when the flankers appeared in the near condition $\left(0.3^{\circ}\right.$ of visual angle from the target). However, this effect was eliminated when the flankers appeared $5^{\circ}$ of visual angle from the target (far condition). This pattern of findings was the same for both same-category (letters) and differentcategory (digits) flankers. Under the no-cue condition, the flanker validity effect occurred for near-digit flankers, near-letter flankers, and far-letter flankers, whereas the fardigit flankers did not result in a significant flanker validity effect. Paquet and Craig therefore concluded that in low-load displays, distractors may not always automatically capture attention. Paquet (2001) also found with lowload displays that interference and negative priming did not occur when the target location was precued and the distractor appeared $2.7^{\circ}$ of visual angle from the target.

Selective attention in low-load displays has also been demonstrated by Johnson, McGrath, and McNeil (2002). Similar to Paquet and Craig (1997), they used cues to focus participants' attention. However, instead of manipulating the flanker validity effect, they examined interference effects. In their experiment, either a central cue always pointed to the target location (100\% valid) or no cue appeared. They also manipulated perceptual load with low- and high-load trials. During a low-load display with a $100 \%$ valid cue, interference effects were not observed, whereas during the no-cue, low-load condition, interference effects did occur. As for the high-load conditions (cue and no-cue), significant interference effects did not occur. Johnson et al. (2002) concluded that their "data suggest that early selection occurred in a low-load situation (the low-load, valid-cue condition), and this finding is problematic for the strong form of the perceptualload hypothesis" (p. 287).

Previous research has solely used cues to focus participants' attention and facilitate selective processing in low-load displays. This is quite different from the original tests of the perceptual load hypothesis (Lavie, 1995), in which cues were never used. The aim of the present research was to test whether selective processing could occur in low-load displays under conditions more similar to Lavie's original studies. This was achieved by manipulating whether distractors were presented as "onsets" or "offsets." Abrupt onsets have been shown to automatically capture attention (e.g., Atchley, Kramer, \& Hillstrom, 2000; Kramer \& Hahn, 1995; Müller \& Rabbitt, 1989; Yantis \& Jonides, 1990). In other words, visual attention is highly sensitive to the abrupt appearance of visual stimuli in a display (Theeuwes, 1991). The automatic capture of attention results in the drawing of visual attention to the location of the abrupt-onset stimulus. Hence, more efficient processing of stimuli located near the abrupt onset should occur in comparison with stimuli located farther away from the abrupt onset (e.g., Theeuwes, 1991; Yantis \& Jonides, 1984).

Subsequent research, however, has found more subtle effects. For example, the contingent involuntary orienting hypothesis (Folk, Remington, \& Johnston, 1992) predicts that when the spatial location of the target stimulus is uncertain, a stimulus will attract attention if the target is also defined by the same property. This is because higher level processes will create an "attention control setting," which allows attention to be captured by events that share the same critical property that defines the target stimulus. This holds true even though the stimulus does not provide information as to the target location. Whether or not a stimulus will capture attention is contingent on that stimulus sharing a feature property of the target stimulus. In other words, if participants are looking for an onset target, they will form an attentional control setting, which allows irrelevant abrupt-onset precues or distractors to involuntarily capture attention. Consistent with the contingent involuntary orienting hypothesis, Folk and colleagues demonstrated that an onset precue captured attention when the target also appeared as an onset. Furthermore, when the target was defined by color, attention was captured by color precues and not by onset precues.

Further support for the contingent involuntary orienting hypothesis comes from experiments by Atchley et al. (2000), which examined whether an onset or offset precue could capture attention, depending on whether the target was presented as an onset or an offset. In their experiments, participants foreknew the type of target presentation (onset or offset). As predicted by the contingent involuntary orienting hypothesis, offset precues only attracted attention when the target was also pre- 
sented as an offset. Furthermore, when the target appeared as an onset, only onset precues attracted attention. Importantly, offset precues had no effect when the target appeared as an onset.

\section{The Present Study}

Our view of the literature to date is that many of the empirical effects attributed to perceptual load may actually be produced by the salience of the distractor and not the perceptual load per se. The salience of the distractor is determined to a large extent by attentional control settings. Thus, the present research set out to investigate the effect of target and distractor presentation on selective processing by examining the effect of distractor presentation (onset vs. offset) on interference in both low- and high-load displays. Two competing hypotheses were tested: the perceptual load hypothesis and the alternative "salience" hypothesis.

According to the perceptual load hypothesis, the type of distractor presentation (onset or offset) should have little effect on whether interference occurs for both lowand high-load conditions. This is because in a low-load display, all the items in the display should be processed, since there are sufficient attentional resources. Therefore, whether the distractor item is presented as an onset or offset should not matter, because there will always be enough attentional resources to fully process and identify the distractor item. However, in a high-load display, all of one's attentional resources are exhausted with the processing of the target and neutral items, leaving no attentional resources to process the distractor item. Thus, significant interference should occur for distractor onset and distractor offset conditions in the low-load displays, but not for any of the high load displays.

The salience hypothesis suggests that it may not be the load of the display, but rather the salience of the distractor item, that results in a significant interference effect. Thus, perceptual load may affect interference not because of influencing capacity, but rather because of changing the saliency of the distractors. If this is the case, other factors influencing saliency should also determine interference. Thus, this hypothesis predicts that distractor onsets should produce significant interference effects under lowload conditions, since a single distractor onset should be highly salient given the attentional control setting for onset targets. The magnitude of interference should be reduced, however, under high-load conditions, because a distractor is naturally less salient when it is presented with several other onsets (target and several neutral items). Distractor offsets, however, should reduce the saliency of the distractor item, since targets are always presented as onsets, thereby enabling participants to engage in selective attention such that the distractor is not processed (e.g., Theeuwes, 1991). The salience hypothesis therefore predicts that the magnitude of interference will be significantly reduced or eliminated during the distractor-offset condition in both low- and high-load displays. The critical test is with the distractor-offset, low-load displays in which interference effects should be eliminated or reduced, because the distractor item is now less salient, even though perceptual load is low.

\section{EXPERIMENT 1}

In the present experiment, we set out to determine what role, if any, the nature of distractor presentation plays in selective target processing. Is it possible to obtain selective attention during a low-load display just by manipulating the saliency of the distractor object? In order to address this question, distractor onsets and offsets were presented while interference effects were measured in a task similar to Lavie's (1995) original perceptual load studies. The extent of distractor processing will be evident by significant interference effects or the lack thereof. Targets were always presented as onsets.

The perceptual load hypothesis predicts that interference will only occur for low-load displays, but not highload displays, regardless of distractor presentation. However, the salience hypothesis predicts that interference will occur for the distractor-onset, low-load condition, but will be significantly reduced or eliminated in the distractoroffset conditions. Interference in the high-load condition should also be reduced, especially in the offset condition, given the reduction in distractor salience.

\section{Method}

Participants. Twenty-seven undergraduate and graduate students from the University of Essex with normal or corrected-to-normal vision participated in this experiment. Three participants were removed from further analysis due to an error rate of more than 2.5 standard deviations from the mean. Of the remaining participants, there were 15 females and 9 males with an average age of 25.75 years $(S D=$ 4.46). The participants were paid $£ 2.50$ for participating.

Apparatus and Stimuli. Stimulus presentation and data collection were controlled by a MEL2 program (Schneider, 1988) on an IBM-PC compatible computer with a VGA color monitor. A custombuilt "viewer" was used to maintain a constant viewing distance of approximately $56 \mathrm{~cm}$ from the participants' eyes to the computer screen. The target letters were "s" and "c." The letters "v," "w," "z," "r" and " $n$ " and the asterisk $(*)$ symbol composed the neutral stimuli. The target appeared in a circular array with either five asterisks (low load) or the five neutral letters (high load). The target letters, asterisk, and neutral letters were $0.5 \mathrm{~cm}$ wide and $0.5 \mathrm{~cm}$ high $\left(0.5^{\circ} \times 0.5^{\circ}\right.$ of visual angle $)$. The target and neutral items appeared $0.6 \mathrm{~cm}\left(0.6^{\circ}\right.$ of visual angle $)$ from the central fixation point and $0.6 \mathrm{~cm}\left(0.6^{\circ}\right.$ of visual angle $)$ from each other. The closest edge of the target or neutral items was $1.0 \mathrm{~cm}\left(1.0^{\circ}\right.$ of visual angle $)$ from the nearest edge of the distractor location box. The distractor letters could either be incompatible ("s" when the target was "c" or vice versa) or neutral (" $p$ " and " $\mathrm{j}$," which only appeared as distractor letters and not as target letters). The width of the distractor letters and figure eights was $1.0 \mathrm{~cm}$ whereas the height was $1.1 \mathrm{~cm}\left(1.0^{\circ} \times\right.$ $1.1^{\circ}$ of visual angle). The target and neutral items were presented in the system font, whereas the figure eight and distractor items were composed of connecting line segments. Distractor location boxes surrounding the distractor items were $2.2 \mathrm{~cm}$ wide and $2.1 \mathrm{~cm}$ high $\left(2.2^{\circ} \times 2.1^{\circ}\right.$ of visual angle). The distractor could appear either to the right or left of the circular array, with its closest edge being $2.5 \mathrm{~cm}\left(2.5^{\circ}\right.$ of visual angle $)$ from the central fixation point. The distance between the distractor item and the nearest target or neutral item was $1.5 \mathrm{~cm}\left(1.5^{\circ}\right.$ of visual angle). In addition, the distance 
between the distractor and the distractor location box was $0.6 \mathrm{~cm}$ to the left and right and $0.5 \mathrm{~cm}$ above and below $\left(0.6^{\circ} \times 0.5^{\circ}\right.$ of visual angle).

Design. Perceptual load, compatibility, and distractor presentation were within-subjects factors. The two levels of perceptual load were low load and high load. In the low-load condition, the target letter appeared with five asterisks. In the high-load condition, the target appeared among five neutral letters ("v," "w," "z," "r," and "n"). Low- and high-load displays were presented in different blocks, and ordering of the presentation of low- and high-load blocks was counterbalanced across participants. The two levels for compatibility were incompatible and neutral, and these were randomly presented in all blocks. In the incompatible condition, the other possible target letter appeared at one of the distractor locations. In the neutral condition, a nontarget letter appeared at one of the possible distractor locations. The two levels for distractor presentation were onset and offset. In the onset condition, the distractor letter appeared simultaneously with the presentation of the target and neutral items. In the offset condition, a block figure eight was presented in the distractor location along with the fixation point. When the target and neutral items were presented, the figure eight became a distractor letter by the removal of line segments (see Figure 1). The two dependent variables were RT and error rates. Interference was measured by the difference in RT between the neutral and incompatible conditions.

Procedure. The participants were given verbal instructions and practice trials before the main experiment. They were told that this was a letter-identification task in which they were to identify the letters " $s$ " and "c," which appeared in a circular array around the central fixation point and to ignore any letters that appeared in the distractor location boxes. At the beginning of each trial, a fixation dot appeared for $1,000 \mathrm{msec}$ at the center of the computer screen. For the offset condition, a block figure eight appeared inside a box at the distractor location along with the central fixation dot. For the onset condition, an empty distractor location box appeared at the distractor location along with the central fixation dot. Each fixation display was followed by the stimulus display for $100 \mathrm{msec}$. The target letters were "s" and "h," and the participants responded by pressing the 1 and 2 keys on the keypad. If the participant made an incorrect response, the computer made a beep sound for $500 \mathrm{msec}$. After the participant responded, a blank screen appeared for $350 \mathrm{msec}$, followed by the fixation dot, distractor location box, and block figure eight (for the offset condition only) for $1,000 \mathrm{msec}$, starting the next trial. The participants were instructed to make their responses as quickly and as accurately as possible.

The total number of experimental trials was 960 , with 480 trials for each of the distractor-onset and -offset conditions. Thirty practice trials preceded each of the distractor presentation $\times$ load conditions: onset low load, onset high load, offset low load, and offset high load. Both low- and high-load conditions had 240 trials, with 120 incompatible and 120 neutral trials. The entire session took approximately $45 \mathrm{~min}$.

\section{Results}

Reaction time. Trials that contained errors or had an RT faster than $200 \mathrm{msec}$ or slower than 1,500 msec were not analyzed. Three percent of the data were lost due to data trimming. A 2 (distractor presentation) $\times 2$ (compatibility) $\times 2$ (load) within-subjects analysis of variance (ANOVA) was performed on the remaining data. Mean RTs and error rates are listed in Table 1. This resulted in a significant main effect for load $[F(1,23)=$ $106.29, p<.001]$ and compatibility $[F(1,23)=6.37$, $p<.05]$. The RTs for the high-load condition were slower than RTs for the low-load condition. The neutral condition resulted in faster RTs than did the incompatible condition. The main effect for distractor presentation was not significant $[F(1,23)=1.22]$.

The two-way interaction between distractor presentation and compatibility was significant $[F(1,23)=5.16$, $p<.05]$. Simple main effects comparisons revealed a significant interference effect for the distractor-onset condition $[F(1,23)=17.24, p<.01]$ but not the distractoroffset condition $[F(1,23)<1]$. The distractor presentation $\times$ load $[F(1,23)<1]$ and the load $\times$ compatibility $[F(1,23)=1.82]$ interactions were not significant. However, the three-way interaction among distractor presentation, load, and compatibility $[F(1,23)=4.94, p<.05]$ was significant.

In order to clarify the three-way interaction among load, compatibility, and distractor presentation, separate

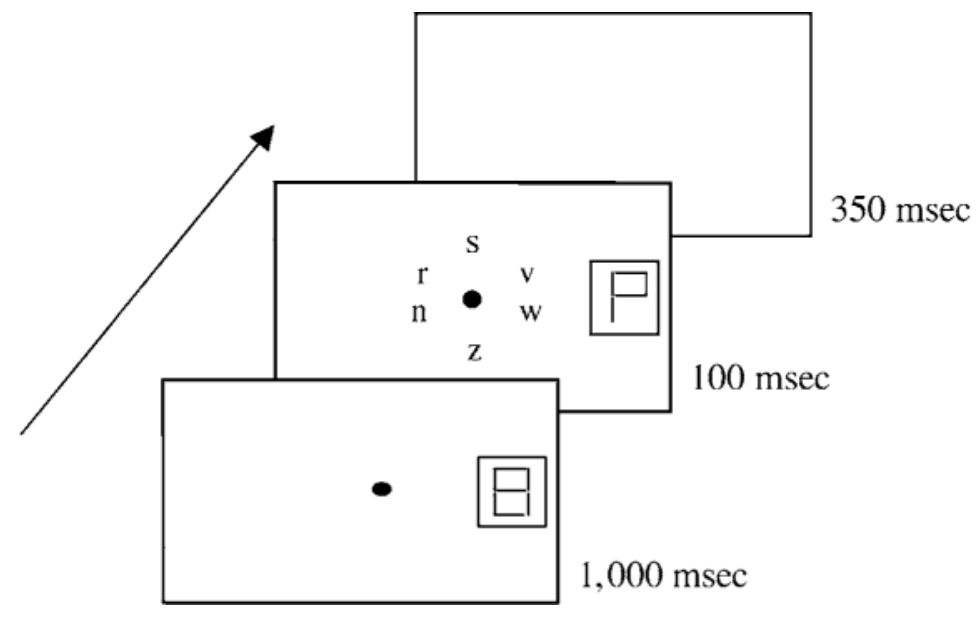

Figure 1. Trial sequence for the distractor-offset, neutral high-load condition in Experiment 1. 
Table 1

Mean Reaction Times (in Milliseconds) and Error Rates for Incompatible and Neutral Onset and Offset Distractors for Low- and High-Load Conditions in Experiment 1

\begin{tabular}{lccccccc}
\hline & \multicolumn{3}{c}{ Low Load } & & \multicolumn{3}{c}{ High Load } \\
\cline { 2 - 3 } \cline { 6 - 7 } & $M$ & $S D$ & $\% \mathrm{E}$ & & $M$ & $S D$ & $\% \mathrm{E}$ \\
\hline Onset & & & & & & & \\
$\quad$ Incompatible & 461 & 63 & 3 & & 575 & 117 & 6 \\
$\quad$ Neutral & 444 & 64 & 3 & & 575 & 117 & 6 \\
Offset & & & & & & \\
$\quad$ Incompatible & 436 & 72 & 3 & & 570 & 90 & 6 \\
$\quad$ Neutral & 439 & 79 & 3 & & 571 & 86 & 6 \\
\hline
\end{tabular}

Note- $\%$ E, error rate.

two-way within-subjects ANOVAs were performed for the distractor-onset and -offset conditions. A 2 (load) $\times 2$ (compatibility) within-subjects ANOVA for the distractoronset condition revealed significant main effects for load $[F(1,23)=59.77, p<.001]$ and compatibility $[F(1,23)=$ $14.34, p<.001]$. Low-load trials had faster RTs than did high-load trials. Incompatible trials resulted in slower RTs than did neutral trials. As expected, there was also a significant interaction between load and compatibility $[F(1,23)=4.68, p<.05]$. Planned $t$ tests revealed a significant compatibility effect for the low load condition $[t(23)=4.31, p<.001]$ but not for the high load condition $[t(23)=1.25]$.

A 2 (load) $\times 2$ (compatibility) within-subject ANOVAs for the distractor-offset condition revealed a significant main effect for load $[F(1,23)=114.72, p<.001]$. Highload trials resulted in slower RTs than did low-load trials. However, the main effect for compatibility and the interaction between load and compatibility were not significant $[F(1,23)<1]$.

Error rate. A 2 (distractor presentation) $\times 2$ (load) $\times$ 2 (compatibility) within-subjects ANOVA was performed on the error data, resulting in a significant main effect for load $[F(1,23)=13.11, p<.01]$. More errors occurred during the high-load condition than in the low-load condition. Neither the main effect for distractor presentation nor compatibility was significant $[F(1,23)<1]$, nor were any of the interactions significant.

\section{Discussion}

Experiment 1 produced a significant three-way interaction among distractor presentation, load, and compatibility. The load $\times$ compatibility interaction in the onset condition replicates previous findings (e.g., Johnson et al., 2002; Lavie, 1995; Lavie \& Cox, 1997; Lavie \& Fox, 2000) and supports the perceptual load hypothesis. However, no main effect for compatibility, or load $\times$ compatibility interaction was found in the offset condition, which supports the salience hypothesis. In particular, a significant interference effect was observed for the distractor-onset, low-load condition, but not for any of the other conditions. This supports the salience hypothesis in that only the salient distractor onset in the lowload condition captured attention, whereas the distractor offset in the same condition did not result in interference. This raises problems for the perceptual load hypothesis, because there should be enough attentional resources in the distractor-offset, low-load display, as evidenced by the significant interference effect in the distractor-onset condition, to allow processing of the distractor item.

\section{EXPERIMENT 2}

A second experiment was conducted to not only replicate the nonsignificant interference effect during the distractor-offset, low-load condition, but also to determine what effect, if any, target salience has on distractor processing. This was accomplished by increasing the size of the target so that it was larger in height $(0.6 \mathrm{~cm})$ than the neutral items $(0.5 \mathrm{~cm})$.

The perceptual load hypothesis claims that the perceptual load of the display, and not target or distractor salience, will determine whether interference effects occur. In other words, interference is the direct result of the perceptual load of the display, and any changes in target or distractor salience will have no effect on whether interference occurs. Therefore, the perceptual load hypothesis predicts that interference will occur for all lowload conditions, regardless of whether the distractor is presented as an onset or offset, but will not occur for any of the high-load conditions.

In comparison, the salience hypothesis claims that the saliency of the target and distractor items, not necessarily the perceptual load of the display, will determine whether interference effects occur. The salience hypothesis postulates that target and distractor items in typical perceptual load experiments become less salient by increasing the number of neutral items in the display - that is, target and distractor items are more salient in lowload displays than in high-load displays. So, if one is able to decrease the saliency of the distractor in a low-load display, the distractor should be less likely to capture attention, and interference should be reduced. Alternatively, if one is able to increase the salience of both the target and distractor items in a high-load display in comparison with the neutral items, both the target and distractor should automatically capture attention, resulting in interference effects. The salience hypothesis predicts that for the low-load conditions, interference will only be observed when the distractor appears as a salient onset, but not when it appears as a nonsalient offset. During the high-load condition when the now-salient target appears with a distractor onset, these items will be the most salient items in the display; therefore, they should capture attention, resulting in interference effects. However, when the salient target in a high-load display appears with a nonsalient distractor offset, only the salient target will capture attention, and interference effects will not occur.

\section{Method}

Participants. Forty-five undergraduate and graduate students from the University of Essex with normal or corrected-to-normal vision participated in this experiment. One person was removed from 
Table 2

Mean Reaction Times (in Milliseconds) and Error Rates for Incompatible and Neutral Onset and Offset Distractors for Low- and High-Load Conditions in Experiment 2

\begin{tabular}{llllllll}
\hline & \multicolumn{3}{c}{ Low Load } & & \multicolumn{3}{c}{ High Load } \\
\cline { 2 - 5 } \cline { 6 - 8 } & $M$ & $S D$ & $\% \mathrm{E}$ & & $M$ & $S D$ & $\% \mathrm{E}$ \\
\hline Onset & & & & & & \\
$\quad$ Incompatible & 395 & 57 & 5 & & 506 & 79 & 6 \\
$\quad$ Neutral & 378 & 51 & 4 & & 494 & 79 & 6 \\
Offset & & & & & & \\
$\quad$ Incompatible & 377 & 51 & 4 & & 498 & 92 & 5 \\
$\quad$ Neutral & 372 & 42 & 3 & & 497 & 91 & 4 \\
\hline
\end{tabular}

Note-\%E, error rate.

further analysis due to an error rate of more than 2.5 standard deviations from the mean. There were 36 female and 8 male participants, with an average age of 24.6 years $(S D=4.61)$. The participants were either paid $£ 2.50$ or received course credit for participating. Twentytwo participants were randomly assigned to the distractor-onset condition, and 22 were assigned to the distractor-offset condition.

Apparatus and Stimuli. The apparatus and stimuli were the same as in Experiment 1. The only difference was the size of the target letters. Instead of being $0.5 \mathrm{~cm}$ wide and $0.5 \mathrm{~cm}$ high $\left(0.5^{\circ} \times\right.$ $0.5^{\circ}$ of visual angle), the target letters were now $0.5 \mathrm{~cm}$ wide and $0.6 \mathrm{~cm}$ high $\left(0.5^{\circ} \times 0.6^{\circ}\right.$ of visual angle $)$. This made the target letters slightly larger than the neutral items in the circular array. The target letters were now the letters "s" and "h."

Design. The design was the same as in Experiment 1, except that distractor presentation was now presented as a between-subjects variable instead of a within-subjects variable. This means that half of the participants were randomly assigned to the distractor-onset condition, while the other half were assigned to the distractor-offset condition.

Procedure. The procedure was the same as in Experiment 1, except that participants were now asked to determine whether the letter "s" or "h" appeared in a circular array around the central fixation dot. The total number of experimental trials was 480 . This was the same for both the distractor-onset and -offset conditions. Thirty practice trials preceded each of the load conditions: low and high load. Both low- and high-load conditions had 240 trials, with 120 incompatible and 120 neutral trials. The entire session took approximately $30 \mathrm{~min}$.

\section{Results}

Reaction time. Trials that contained errors or had an RT faster than $200 \mathrm{msec}$ or greater than $1,500 \mathrm{msec}$ were not analyzed. One percent of the data was lost due to data trimming. A 2 (distractor presentation) $\times 2$ (compatibility) $\times 2$ (load) mixed design ANOVA was performed on the remaining data. Mean RTs and error rates are listed in Table 2. This resulted in a significant main effect for load $[F(1,42)=166.78, p<.001]$ and compatibility $[F(1,42)=16.23, p<.001]$. The main effect for distractor presentation was not significant $[F(1,42)<1]$. Low-load displays resulted in faster RTs than did highload displays. Incompatible distractors resulted in slower RT than did neutral distractors.

There was a significant two-way interaction between compatibility and distractor presentation $[F(1,42)=$ $8.16, p<.01]$. Simple main effects comparisons revealed a significant interference for the distractor-onset condition $[F(1,42)=23.70, p<.001]$ but not for the distractor-offset condition $[F(1,42)<1]$. Neither the compatibility $\times$ load $[F(1,42)=1.52]$, nor the compatibility $\times$ load $\times$ distractor presentation $[F(1,42)<1]$ interactions were significant.

Error rate. A 2 (distractor presentation) $\times 2$ (load) $\times$ 2 (compatibility) mixed design ANOVA was performed on the error data. The main effects for load $[F(1,42)=11.87$, $p<.001]$ and compatibility $[F(1,42)=4.94, p<.05]$ were significant. The low-load condition resulted in fewer errors than did the high-load condition, and the incompatible condition resulted in more errors than did the neutral condition. The main effect for distractor presentation was not significant, nor were any of the interactions.

\section{Discussion}

A significant interference effect was found for the distractor-onset, low- and high-load conditions, but not for any of the distractor-offset conditions. This supports the salience hypothesis in that interference effects were not observed in either the low- or high-load condition when the distractor appeared as an offset, but they did occur in the conditions in which the target and distractors were most salient. It appears that when the target and distractor are the most salient items in a display, they both automatically capture attention, resulting in interference effects. In comparison, when the distractor appears as an offset it does not automatically capture attention. In fact, participants were able to completely ignore the distractor letter, thereby eliminating the possibility of interference effects.

Neither the compatibility $\times$ load, nor the three-way compatibility $\times$ load $\times$ distractor presentation interactions were significant in this experiment. The magnitude of interference for the distractor-onset, low-load condition was $17 \mathrm{msec}[t(21)=5.70, p<.001]$, whereas the high-load condition was $12 \mathrm{msec}[t(21)=2.43, p<.05]$. These results are contrary to what is predicted by the perceptual load hypothesis, which claims that interference will not occur in high-load displays since there are not enough attentional resources to process the distractor item. However, the salience hypothesis predicts that interference would occur in high-load displays if both the target and distractor were the most salient items in a display, resulting in the automatic capture of attention. It is important to note that the target letter was only slightly taller than the other neutral items: $0.1 \mathrm{~cm}\left(0.1^{\circ}\right.$ of visual angle). Nevertheless, we suspect that the larger size of the target changed the "set" used by participants to locate the target. The set adopted to detect a singleton (large) target might also lead to attention being captured by the onset distractor. The mechanism provides an explanation of the present results, including significant interference in the high-load condition, and is incompatible with the perceptual load hypothesis.

\section{EXPERIMENT 3}

Taken together, results from Experiments 1 and 2 suggest that the saliency of the target and the distractor can determine whether interference effects occur. Across the 
two experiments, the perceptual load is challenged. First, a high-load interference effect was found, and second, the low-load interference effect was eliminated. These results suggest that perceptual load itself does not determine the magnitude of interference effects. Instead, other factors that change concurrently with perceptual load manipulation (e.g., salience of distractor, perceptual set) may determine the size of interference effects. Experiment 3 was conducted to further examine the contribution of attentional set in eliciting distractor interference effects. This was done by manipulating target and distractor presentation so that they could both appear as either an onset or an offset. Target presentation was manipulated between subjects, whereas distractor presentation was manipulated as a within-subjects variable. Only low-load trials were used in this experiment.

The perceptual load hypothesis claims that neither the type of target (onset or offset) nor distractor presentation (onset or offset) should determine interference effects. Since the perceptual load of the displays is always low in the present experiment, the perceptual load theory would predict interference effects for all four conditions: onsettarget, onset-distractor; onset-target, offset-distractor; offset-target, offset-distractor; and offset-target, onsetdistractor.

The contingent involuntary orienting hypothesis, however, claims that individuals are able to develop an attentional control setting based on the defining property of the target (e.g., onset, color, movement) that only allows stimuli sharing that critical property to capture attention. Given this, participants should be able to develop an attentional control setting that allows all onset stimuli to capture attention when the target appears as an onset and an attentional control setting that allows all offset stimuli to capture attention when the target appears as an offset. In addition, offset distractors should not capture attention when the target appears as an onset, and onset distractors should not capture attention when the target appears as an offset. Thus, the contingent involuntary orienting hypothesis would predict interference effects for the targetonset, distractor-onset and target-offset, distractor-offset conditions. However, interference effects should not occur for the target-onset, distractor-offset or target-offset, distractor-onset conditions.

The salience hypothesis claims that the saliency of the target and distractor determines whether interference effects occur. Since salience is determined to a large extent by attentional set, when the distractor is an onset, it will be most salient when the target is presented as an onset. However, when the distractor is an offset, it will only be salient when the target is also presented as an offset. The salience hypothesis predicts that interference effects will occur for the target-onset, distractor-onset and targetoffset, distractor-offset conditions. Interference effects should be reduced or eliminated in the target-offset, distractor-onset and target-onset, distractor-offset conditions. The perceptual load hypothesis predicts interference effects in all the conditions since they are low load.

\section{Method}

Participants. Forty-four undergraduate and graduate students from the University of Essex with normal or corrected-to-normal vision were used in this experiment. There were 29 female and 15 male participants, with an average age of 26.2 years $(S D=3.48)$. They were paid $£ 2.50$ for participating. Twenty-two of the participants were randomly assigned to the target-onset condition, and 22 were assigned to the target-offset condition.

Apparatus and Stimuli. The apparatus and stimuli were the same as in Experiment 1, except for the few changes mentioned below. The neutral items in the circular array were no longer the asterisk symbol, but were three horizontal lines (三). For the target-offset condition, six figure eights appeared in a circular array around the central fixation point and became the target and neutral items by the removal of line segments. The target letters, neutral items, and figure eights were $0.5 \mathrm{~cm}$ wide and $0.5 \mathrm{~cm}$ high $\left(0.5^{\circ} \times 0.5^{\circ}\right.$ of visual angle $)$.

Design. Distractor presentation and compatibility were manipulated as within-subjects variables, whereas target presentation was a between-subjects variable. The two levels of distractor presentation were onset and offset. In the distractor-onset condition, the distractor appeared in the distractor location box along with the presentation of the target. In the distractor-offset condition, a block figure eight was presented at the distractor location along with a distractor location box and central fixation point. In conjunction with the presentation of the target, the figure eight became the distractor letter by the removal of line segments. The presentation of the distractor-onset and the distractor-offset conditions was counterbalanced across participants with half receiving the distractor-onset followed by distractoroffset presentation, and the other half receiving the distractor-offset followed by distractor-onset presentation. The two levels of compatibility were incompatible and neutral. In the incompatible condition, the other possible target letter appeared as the distractor. In the neutral condition, a nontarget letter appeared as the distractor. Both incompatible and neutral trials were randomly presented across the experiment. The two levels of target presentation were onset and offset. In the target-onset condition, the target and neutral items appeared suddenly on the screen. In the target-offset condition, six figure eights appeared in a circular array around the central fixation point. The figure eights became the target and neutral items by the removal of line segments (see Figure 2). The two dependent variables were RTs and error rates. Interference was measured by the difference in RT between the neutral and incompatible trials.

Procedure. The procedures were the same as in Experiments 1 and 2. The only difference between the procedures used in Experiments 1 and 2 and those used in Experiment 3 is the fixation display used for offset targets and the appearance of target-offset and neutral items. In the target-offset, distractor-offset condition, the central fixation dot, six block figure eights in a circular array, a distractor location box, and a block figure eight inside the distractor location box were presented during the fixation display. Then the target, neutral, and distractor items were simultaneously presented through the removal of line segments. In the target-offset, distractoronset condition, the central fixation dot, six block figure eights, and a distractor location box appeared as the fixation display. As for the presentation of the stimulus display, the target and neutral items appeared through the removal of line segments, whereas the distractor simultaneously appeared in the distractor location box.

The total number of experimental trials was 480 . This was the same for the target-onset and target-offset conditions. Thirty practice trials preceded each of the distractor presentation conditions: onset and offset. There were 240 distractor-onset and -offset trials, with 120 incompatible and 120 neutral trials. The entire experiment took approximately $30 \mathrm{~min}$.

\section{Results}

Reaction time. Trials that contained errors or had RTs faster than $200 \mathrm{msec}$ or slower than $1,500 \mathrm{msec}$ were not 


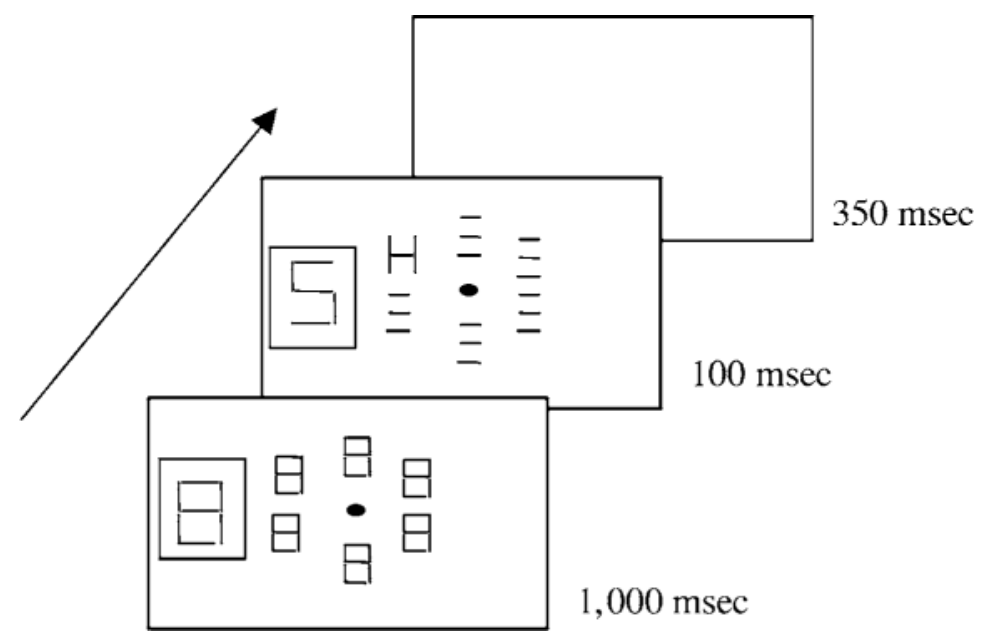

Figure 2. Trial sequence for the target-offset, distractor-offset incompatible condition in Experiment 3.

analyzed. Less than $1 \%$ of the data was lost through data trimming. A 2 (target presentation) $\times 2$ (distractor presentation) $\times 2$ (compatibility) mixed design ANOVA was performed. Mean RTs and error rates are presented in Table 3. The main effects for distractor presentation $[F(1,42)=15.58, p<.01]$, compatibility $[F(1,42)=$ $21.87, p<.01]$, and target presentation $[F(1,42)=15.41$, $p<.01]$ were significant. Distractor offsets resulted in faster RTs than did distractor onsets. Incompatible trials resulted in slower RTs than did neutral trials. Target offsets resulted in slower RTs than did target onsets.

The interaction between compatibility and target presentation was significant $[F(1,42)=8.04, p<.01]$. Simple main effects comparisons revealed a significant interference effect for the target-offset condition $[F(1,42)=$ $28.21, p<.01]$, but not for the target-onset condition $[F(1,42)<1.71]$. In addition, as in the previous experiments the interaction between compatibility and distractor presentation was significant $[F(1,42)=19.03, p<.01]$. Simple main effects comparisons resulted in a significant compatibility effect for the distractor-onset condition $[F(1,42)=40.84, p<.01]$, but not for the distractoroffset condition $[F(1,42)<1]$. The two-way interaction between distractor and target presentation was not significant $[F(1,42)=1.01, p>.05]$, nor was the three-way interaction among distractor presentation, target presentation, and compatibility $[F(1,42)<1]$.

Given the a priori predictions, paired-samples $t$ tests were performed to determine whether interference effects occurred for each of the four conditions: targetonset, distractor-onset; target-onset, distractor-offset; target-offset, distractor-offset; and target-offset, distractoronset. This revealed that when the target was an onset, only onset distractors $[t(22)=3.86, p<.01]$ resulted in a significant interference. Offset distractors showed no interference effects $[t(22)=-1.03, p>.05]$, supporting the salience hypothesis. Contrary to the salience hypothesis, when the target appeared as an offset, both onset dis- tractors $[t(22)=4.72, p<.01]$ and offset distractors $[t(22)=1.90, p<.05]$ showed significant interference effects.

Error rate. A 2 (target presentation) $\times 2$ (distractor presentation) $\times 2$ (compatibility) mixed design ANOVA was performed on the error data. The main effects for compatibility $[F(1,42)=9.08, p<.01]$ and target presentation $[F(1,42)=4.65, p<.05]$ were significant. More errors occurred during the incompatible than during the neutral trials. Fewer errors occurred in the target-onset than in the target-offset condition. The main effect for distractor presentation showed a trend $[F(1,42)=3.77, p=$ $.059]$, with fewer errors occurring in the distractor-offset than in the distractor-onset condition. None of the interactions were significant.

\section{Discussion}

The failure to find a significant three-way interaction (distractor presentation $\times$ target presentation $\times$ compatibility) implies that the manipulation of target presentation (onset vs. offset) was not entirely effective in changing attentional control settings. Nevertheless, the significant two-way interaction between distractor presentation and

Table 3

Mean Reaction Times (in Milliseconds) and Error Rates for Incompatible and Neutral Onset and Offset Distractors and Targets in Experiment 3

\begin{tabular}{|c|c|c|c|c|c|c|}
\hline \multirow[b]{3}{*}{ Distractor Presentation } & \multicolumn{6}{|c|}{ Target Presentation } \\
\hline & \multicolumn{3}{|c|}{ Onset } & \multicolumn{3}{|c|}{ Offset } \\
\hline & $M$ & $S D$ & $\% \mathrm{E}$ & $M$ & $S D$ & $\% \mathrm{E}$ \\
\hline \multicolumn{7}{|l|}{ Onset } \\
\hline Incompatible & 429 & 80 & 3 & 526 & 91 & 6 \\
\hline Neutral & 419 & 78 & 3 & 506 & 91 & 4 \\
\hline \multicolumn{7}{|l|}{ Offset } \\
\hline Incompatible & 396 & 53 & 3 & 475 & 78 & 4 \\
\hline Neutral & 400 & 57 & 2 & 470 & 77 & 4 \\
\hline
\end{tabular}

Note-\%E, error rate. 
compatibility fits the salience hypothesis better than the perceptual load hypothesis. As in Experiments 1 and 2, when the target was presented as an onset, only distractor onsets resulted in interference effects, whereas distractor offsets did not. The perceptual load hypothesis is unable to explain why interference was not found when the target appeared as an onset and the distractor appeared as an offset. Given that it was a low-load condition and interference was found in the distractor-onset condition, there should be enough attentional resources in the distractor-offset condition to process both the target and distractor items, hence interference should occur. The most logical explanation seems to be that the distractor was not salient in the offset condition, which enabled participants to selectively attend to the target letter while ignoring the distractor letter and thereby not processing the distractor letter. Thus, selective attention can occur in low-load displays even in the absence of a precue to target locations.

Experiment 3 also showed that when the target was presented as an offset, both onset and offset distractors resulted in interference. This result does not support the salience hypothesis in that participants in the target-offset condition should have been able to develop an attentional control setting that allowed stimulus offsets but not stimulus onsets to automatically capture attention. However, it is possible that the onset distractor was just so salient that it could not be ignored even in the context of cue-contingent orienting.

\section{GENERAL DISCUSSION}

Experiments 1, 2, and 3 provide a conceptual replication and extension of Paquet and Craig (1997), Paquet (2001), and Johnson et al. (2002). Whereas they utilized precues to focus participants' attention, our study manipulated target and distractor presentations (offsets and onsets) in a flanker task. Our task was much closer to the original perceptual load experiments. However, we found that participants were able to engage in focused attention during low-load displays when the distractor was presented as an offset, at least while the target was presented as an onset. This is consistent with the conclusion of $\mathrm{Pa}$ quet and Craig, that "selective target processing is possible with low load displays, and that, under appropriate conditions, the flankers will not capture attention" (p. 186). Importantly, the present experiments show that focused attention is possible without having to precue target location.

The lack of any significant interference effect for the target-onset, distractor-offset presentations during lowload displays is contrary to the perceptual load theory (Lavie, 1995; Lavie \& Tsal, 1994), which claims that attention is an automatic process as long as there are available perceptual resources. Lavie (1995) argues that, "whether selective processing will occur is at the mercy of the perceptual load imposed by external events" (p. 466). In other words, stimulus processing is deter- mined by the stimulus display, and people have little or no control over the allocation of attention.

In contrast to the perceptual load hypothesis, Experiments 1,2 , and 3 supported the hypothesis that primarily the saliency of the target and distractor items determine whether or not a distractor will be processed. This was evident in three different outcomes: Salient targets can result in distractors being processed in high-load displays; distractor offsets do not capture attention when targets are presented as onsets even in low-load displays; and distractor offsets do capture attention when the target also appears as an offset. Two key findings are problematic for the perceptual load theory. First of all, participants could engage in a focused mode of attention during low-load displays, resulting in no interference effects when the distractor was presented as an offset and the target appeared as an onset. Second, interference effects were found during high-load displays just by making the target letter a singleton. These findings provide evidence that converges with those of Paquet and Craig (1997), Paquet (2001), and Johnson et al. (2002) and are contrary to the predictions of the perceptual load hypothesis.

In summary, the present results show that the perceptual load of the display does not seem to be the primary determinant of selective processing. Rather, distractor salience was the most important factor in determining distractor processing. Thus, the usual perceptual load effect may occur because the distractor in a high perceptual load display is less likely to capture attention and not because resources are fully utilized.

\section{REFERENCES}

Atchley, P., Kramer, A. F., \& Hillstrom, A. P. (2000). Contingent capture for onsets and offsets: Attentional set for perceptual transients. Journal of Experimental Psychology: Human Perception \& Performance, 26, 594-606.

Broadbent, D. E. (1958). Perception and communication. New York: Pergamon.

Deutsch, J. A., \& Deutsch, D. (1963). Attention: Some theoretical considerations. Psychological Review, 70, 80-90.

Duncan, J. (1980). The locus of interference in the perception of simultaneous stimuli. Psychological Review, 87, 272-300.

ERIKSEN, B. A., \& ERIKSEN, C. W. (1974). Effects of noise letters upon the identification of a target letter in a nonsearch task. Perception $\&$ Psychophysics, 16, 143-149.

Eriksen, C. W., \& Hoffman, J. E. (1972). Temporal and spatial characteristics of selective encoding from visual displays. Perception $\&$ Psychophysics, 12, 201-204.

Eriksen, C. W., \& Hoffman, J. E. (1973). The extent of processing of noise elements during selective encoding from visual displays. Perception \& Psychophysics, 14, 155-160.

FolK, C. L., Remington, R. W., \& Johnston, J. C. (1992). Involuntary covert orienting is contingent on attentional control settings. Journal of Experimental Psychology: Human Perception \& Performance, 18, 1030-1044.

Johnson, D. N., McGrath, A., \& McNeil, C. (2002). Cuing interacts with perceptual load in visual search. Psychological Science, 13, 284-287.

Kramer, A. F., \& Hahn, S. (1995). Splitting the beam: Distribution of attention over noncontiguous regions of the visual field. Psychological Science, 6, 381-386.

LAVIE, N. (1995). Perceptual load as a necessary condition for selective attention. Journal of Experimental Psychology: Human Perception \& Performance, 21, 451-468. 
Lavie, N. (2000). Selective attention and cognitive control: Dissociating attentional functions through different types of load. In S. Monsell \& J. Driver (Eds.), Control of cognitive processes: Attention and performance XVIII (pp. 175-194). Cambridge, MA: MIT Press.

LAVIE, N., \& Cox, S. (1997). On the efficiency of visual selectivity attention: Efficient visual search leads to inefficient distractor rejection. Psychological Science, 8, 395-398.

LaVIE, N., \& Fox, E. (2000). The role of perceptual load in negative priming. Journal of Experimental Psychology: Human Perception \& Performance, 26, 1038-1052.

LaVIE, N., \& TsaL, Y. (1994). Perceptual load as a major determinant of locus of selection in visual attention. Perception \& Psychophysics, 56, 183-197.

MÜLleR, H.J., \& RabBitT, P.M. A. (1989). Reflexive and voluntary orienting of visual attention: Time course of activation and resistance to interruption. Journal of Experimental Psychology: Human Perception \& Performance, 15, 315-330.

Navon, D., \& Gopher, D. (1979). On the economy of the humanprocessing system. Psychological Review, 86, 214-255.

PAQUET, L. (2001). Eliminating flanker effects and negative priming in the flankers task: Evidence for early selection. Psychonomic Bulletin \& Review, 8, 301-306.
Paquet, L., \& Craig, G. L. (1997). Evidence for selective target processing with a low perceptual load flankers task. Memory \& Cognition, 25, 182-189.

SCHNEIDER, W. (1988). Micro Experimental Laboratory: An integrated system for IBM PC compatibles. Behavior Research Methods, Instruments, \& Computers, 20, 206-217.

Stroop, J.R. (1935). Studies of interference in serial verbal reactions. Journal of Experimental Psychology, 2, 643-662.

TheEUwES, J. (1991). Exogenous and endogenous control of attention: The effect of visual onsets and offsets. Perception \& Psychophysics, 49, 83-90.

Treisman, A., \& Gormican, S. (1988). Feature analysis in early vision: Evidence from search asymmetries. Psychological Review, 95, 15-48.

YANTIS, S., \& Jonides, J. (1984). Abrupt visual onsets and selective attention: Evidence from visual search. Journal of Experimental Psychology: Human Perception \& Performance, 10, 601-621.

YANTIS, S., \& JONIDES, J. (1990). Abrupt visual onsets and selective attention: Voluntary versus automatic allocation. Journal of Experimental Psychology: Human Perception \& Performance, 16, 121-134.

(Manuscript received February 20, 2003; revision accepted for publication October 29, 2004.) 